\title{
S-Nitrosylation of Cyclin-Dependent Kinase 5 (Cdk5) Regulates Its Kinase Activity and Dendrite Growth During Neuronal Development
}

\author{
Peng Zhang, Pei-Chun Yu, Anthony H. K. Tsang, Yu Chen, Amy K. Y. Fu, Wing-Yu Fu, Kenny K. Chung, and Nancy Y. Ip \\ Department of Biochemistry, Molecular Neuroscience Center and State Key Laboratory of Molecular Neuroscience, The Hong Kong University of Science \\ and Technology, Clear Water Bay, Hong Kong, China
}

Precise regulation of cyclin-dependent kinase 5 (Cdk5), a member of the cyclin-dependent kinase family, is critical for proper neuronal development and functions. Cdk5 is activated through its association with the neuron-specific activator $\mathrm{p} 35$ or p39. Nonetheless, how its kinase activity is regulated in neurons is not well understood. In this study, we found that Cdk5 activity is regulated by S-nitrosylation, a post-translational modification of protein that affects a plethora of neuronal functions. S-nitrosylation of Cdk5 occurs at Cys 83 , which is one of the critical amino acids within the ATP-binding pocket of the kinase. Upon S-nitrosylation, Cdk5 exhibits reduced kinase activity, whereas mutation of Cys 83 to Ala on Cdk5 renders the kinase refractory to such inhibition. Importantly, S-nitrosylated Cdk 5 can be detected in the mouse brain, and blocking the S-nitrosylation of Cdk5 in cultured hippocampal neurons enhances dendritic growth and branching. Together, our findings reveal an important role of S-nitrosylation in regulating Cdk5 kinase activity and dendrite growth in neurons during development.

\section{Introduction}

Cyclin-dependent kinase 5 (Cdk5), a proline-directed serine/ threonine kinase, plays important roles in various aspects of neural development including neuronal migration, axon guidance, synapse development, and plasticity (Dhavan and Tsai, 2001; Cheung et al., 2006; Lai and Ip, 2009). Deregulation of Cdk5 activity is linked with the pathophysiological mechanisms of various neurological disorders including Alzheimer's disease and Parkinson's disease (Cruz and Tsai, 2004). Thus, precise regulation of Cdk5 is important for normal development and functions of the nervous system. Nonetheless, the molecular mechanisms underlying the regulation of Cdk5 activity are not well understood.

Unlike other members of the cyclin-dependent kinase family, Cdk5 is not regulated by cyclins, but is activated through its association with neuronal-specific activator $\mathrm{p} 35$ or $\mathrm{p} 39$. Whereas the activity of Cdk5 in neurons is largely determined by the expression and subcellular localization of these activators (Hisanaga and Saito, 2003), the protein level of p35 and p39 in neurons is mainly controlled by a balanced act of protein synthesis and ubiquitin-/proteasomal-dependent protein degradation. Under pathological condition, p35 or p39 undergoes calpain-

Received July 27, 2010; revised Sept. 2, 2010; accepted Sept. 5, 2010.

This study was supported in part by the Research Grants Council of Hong Kong (Grants HKUST 6444/06M, 661007, and 661109), the Area of Excellence Scheme of the University Grants Committee (AoE/B-15/01), and the Hong Kong Jockey Club. N.Y.I. was recipient of the Croucher Foundation Senior Research Fellowship. We are grateful to Dr. Shirley Xie and Miss Cara Kwong for their excellent technical assistance. We also thank members of the Ip laboratory for many helpful discussions.

Correspondence should be addressed to either Kenny K. Chung or Nancy Y. Ip, Department of Biochemistry, The Hong Kong University of Science and Technology, Clear Water Bay, Hong Kong, China, E-mail: BCKCHUNG@UST.HK or BOIP@UST.HK.

DOI:10.1523/JNEUROSCI.3899-10.2010

Copyright $\odot 2010$ the authors $\quad 0270-6474 / 10 / 3014366-05 \$ 15.00 / 0$ dependent cleavage, generating a proteolytic product, p25 or p29 (Kusakawa et al., 2000; Lee et al., 2000; Patzke and Tsai, 2002), which deregulates Cdk5 activity through the mislocalization and mistargeting of Cdk5 to its substrates. On the other hand, phosphorylation of Cdk5 or its activators can also regulate its kinase activity (Saito et al., 2003; Kamei et al., 2007). For example, phosphorylation of Cdk5 at tyrosine 15 (Tyr 15) following stimulation by extracellular cues such as ephrinA-1 has been reported to enhance its kinase activity (Dhavan and Tsai, 2001; Fu et al., 2007).

In addition to protein phosphorylation, emerging evidence has also revealed protein S-nitrosylation, a post-translational modification that involves a reversible coupling of nitric oxide (NO) to the cysteine sulfhydryls, as an important regulatory signaling mechanism (Lipton et al., 1998). Under dynamic regulation by the NO signaling pathway, S-nitrosylation of a protein can modify its activity or function. For example, it has been reported that S-nitrosylation is important for regulating the catalytic activity of various kinases, including $G$ protein-coupled receptor kinase 2, Akt/protein kinase B, Src kinase, inhibitory $\kappa \mathrm{B}$ kinase, or $\mathrm{Ca}^{2+} /$ calmodulin-dependent protein kinase II (Reynaert et al., 2004; Yasukawa et al., 2005; Whalen et al., 2007; Song et al., 2008; Rahman et al., 2010). Whereas NO signaling pathway is one of the key regulators in a wide array of neurodevelopmental processes, including neuronal differentiation, neuronal survival, and synaptic plasticity (Guix et al., 2005), deregulation of this pathway has been implicated in the pathogenesis of neurological disorders such as Alzheimer's disease, Parkinson's disease, and stroke (Chung, 2006).

In this study, we found that Cdk5 is S-nitrosylated at cysteine 83 (Cys83), and this modification suppresses its kinase activity. The Cdk5-C83A mutant, in which the Cys83 residue is replaced 
a

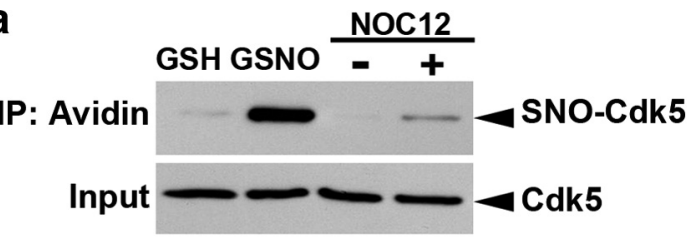

b

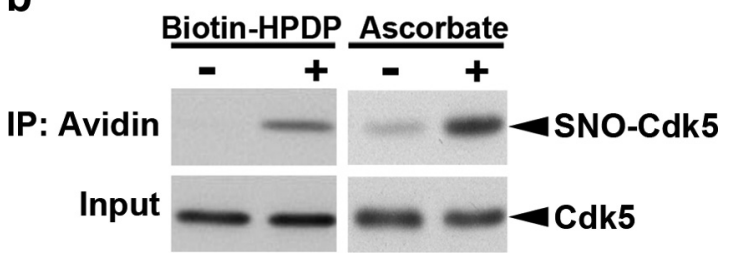

Figure 1. C $\mathrm{dk} 5$ protein is S-nitrosylated in vitro and in vivo. $\boldsymbol{a}, \mathrm{NO}$ S-nitrosylates C $\mathrm{dk} 5$ in vitro. Lysate of HEK 293T cells overexpressing Cdk5 was incubated with NO donor GSNO or NOC12 (GSH or depleted NOC12 as control) for 30 min, and then subjected to biotin switch assay. $\boldsymbol{b}$, (dk5 was $S$-nitrosylated in adult rat brain. Adult mouse brain lysate was subjected to the biotin switch assay in the presence of biotin-HPDP or ascorbate as indicated. The biotinylated proteins were immunoprecipitated (IP) with Neutravidin-agarose, followed by Western blot analysis for Cdk5.

a

Amino acid sequence of mouse Cdk5

$\begin{array}{lll}1 & 31 \\ \text { MQKYEKLEKI GEGTYGTVFK AKNRETHEIV } & \text { ALKRVRLDDD DEGVPSSALR EICLLKELKH } \\ 61 & 91 & \\ \text { KNIVRLHDVL HSDKKLTLVF EFCDQDLKKY } & \text { FDSCNGDLDP EIVKSFLFQ LKGLGFCHSR } \\ 121 & 151 \\ \text { NVLHRDLKPQ NLLINRNGEL KLADFGLARA } & \text { FGIPVRCYSA EVVTLWYRPP DVLFGAKLYS } \\ 181 & 211 & \\ \text { TSIDMWSAGC IFAELANAGR PLFPGNDVDD } & \text { QLKRIGRLLG TPTEEQWPAM TKLPDYKPYP } \\ 241 & 271 & \\ \text { MYPATTSLVN VVPKLNATGR DLLQNLLKCN } & \text { PVQRISAEEA LQHPYFSDFC PP }\end{array}$

b
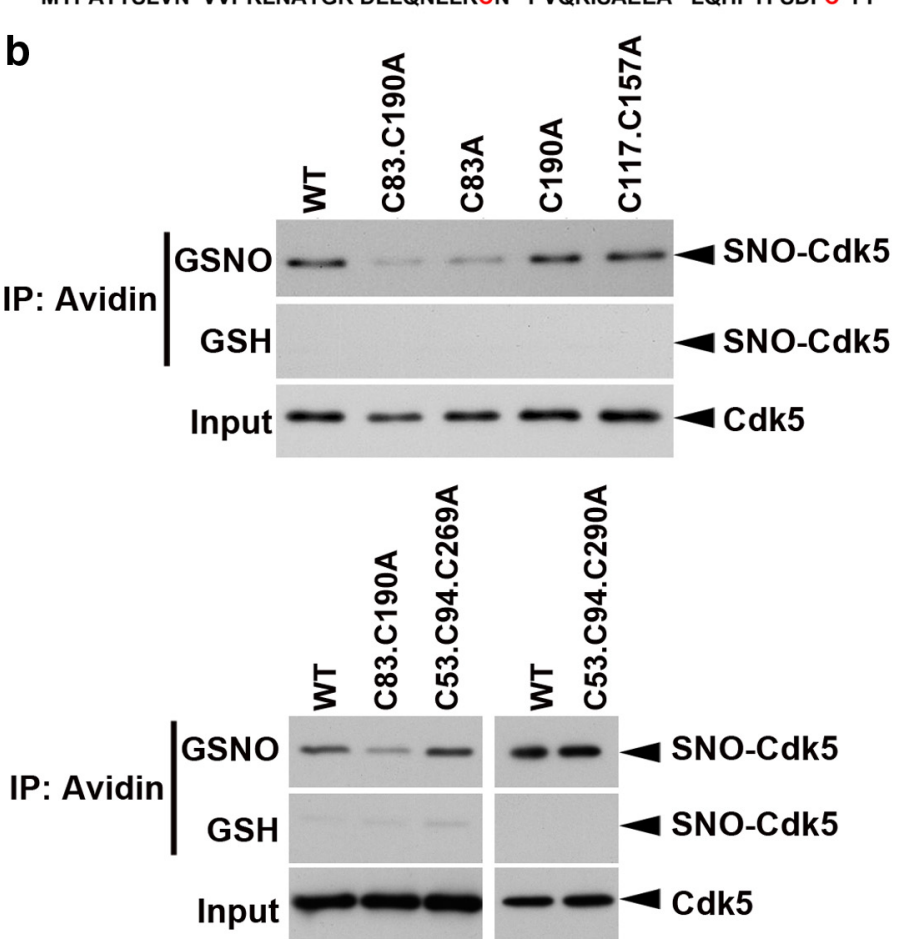

Figure 2. Cys 83 residue of $C \mathrm{dk} 5$ is the target site for S-nitrosylation. $\boldsymbol{a}$, Amino acid sequence of mouse C $\mathrm{dk} 5$ protein. The eight cysteine residues, which are the potential S-nitrosylation sites, were denoted in red color. $\boldsymbol{b}$, HEK 293T cells were transfected with expression constructs encoding the WT or specific cysteine mutants of ( $\mathrm{dk} 5$ as indicated. The cell lysate was then incubated with GSH or GSNO for $30 \mathrm{~min}$ and then subjected to biotin switch assay. The biotinylated proteins immunoprecipitated (IP) with Neutravidin-agarose were subjected to Western blot analysis for Cdk5.

by Ala, is resistant to the NO donor-induced S-nitrosylation and, as such, renders the kinase refractory to the inhibition. Interestingly, perturbing the S-nitrosylation of Cdk5 at Cys83 enhances dendrite development in cultured hippocampal neurons, impli- cating a potential role of S-nitrosylated Cdk5 in the regulation of neuronal development.

\section{Materials and Methods}

Chemicals and antibodies. All chemicals were purchased from Sigma unless otherwise stated. Antibodies specific for Cdk5 (C-8 and DC-17) and p35 (C-19) antibodies were purchased from Santa Cruz Biotechnology.

Site-directed mutagenesis. Site-directed mutagenesis was performed with oligonucleotide primers that were designed to substitute the corresponding cysteine residue(s) with alanine residue(s) using the QuikChange SiteDirected Mutagenesis Kit (Stratagene).

In vitro S-nitrosylation assay. The biotin switch assay for detecting the S-nitrosylation was performed as previously described (Tsang et al., 2009). Adult male mouse brain or HEK 293T cells overexpressing Cdk5 or its cysteine mutants were lysed in HENT buffer (250 mM HEPES, $1 \mathrm{~mm}$ EDTA, $0.1 \mathrm{~mm}$ neocuproine, $1 \%$ Triton X-100). Cell lysates were incubated with $10 \mathrm{~mm}$ methyl methanethiosulfonate (MMTS) (Thermo Scientific) at $50^{\circ} \mathrm{C}$ for $20 \mathrm{~min}$, and excess MMTS was removed by passing through the G25 Sephadex spin column three times. The samples were then incubated with $5 \mathrm{~mm}$ ascorbate and $0.4 \mathrm{~mm} \mathrm{~N}$-[6-(biotinamido)hexyl]-3pyridyldithio)-propionamide (biotin-HPDP) (Thermo Scientific) at room temperature with rotation for $1 \mathrm{~h}$. Unreacted biotin-HPDP was removed by G25 Sephadex spin column, and the biotinylated samples were then incubated with 50 $\mu \mathrm{l}$ of Neutravidin-agarose (Thermo Scientific) for $1 \mathrm{~h}$. Pellets were then washed five times with neutralization buffer (20 mM HEPES, pH 8.0, 100 mm NaCl, 1 mm EDTA, 0.5\% Triton X-100) with $0.6 \mathrm{M} \mathrm{NaCl}$, eluted by SDS sample buffer and subjected to Western blot analysis.

Fusion protein and in vitro kinase assay. Glutathione $S$-transferase (GST)-recombinant proteins encoding Cdk5 or its cysteine mutant (C83A) were expressed in the Escherichia coli BL21 strain and purified using a glutathioneSepharose 4B column. In vitro Cdk5/p35 kinase assay was performed as previously described (Fu et al., 2007). In brief, recombinant Cdk5/p35 (Invitrogen) or immunoprecipitated Cdk5/p35 was preincubated with $500 \mu \mathrm{M}$ glutathione $(\mathrm{GSH})$ or $S$-nitrosoglutathione (GSNO) at room temperature for $30 \mathrm{~min}$ with shaking. The Cdk5/p35 kinase assay was then performed in the kinase buffer [ $20 \mathrm{~mm} 3-(\mathrm{N}-$ morpholino)-propanesulfonic acid, $\mathrm{pH} 7.4$, $15 \mathrm{mM} \mathrm{MgCl}_{2}, 100 \mu \mathrm{M}$ ATP] containing 1-2 $\mu \mathrm{Ci}$ of $\left[\gamma^{-32} \mathrm{P}\right] \mathrm{ATP}$ and $8 \mu \mathrm{g}$ of histone-H1 protein at $30^{\circ} \mathrm{C}$ for $30 \mathrm{~min}$. The phosphorylated histone-H1 protein was then separated using $15 \%$ SDS-PAGE and visualized by autoradiography. The band intensity was quantified using the ImageJ software (National Institutes of Health).

Cell cultures and transfection. HEK 293T cells were cultured in DMEM (Invitrogen) supplemented with $10 \%$ heat-inactivated fetal bovine serum plus antibiotics. Primary hippocampal neurons and cortical neurons were prepared from embryonic day 18-19 rat embryos, seeded on cultured plates coated with poly-Dlysine $(50 \mu \mathrm{g} / \mathrm{ml})$ and maintained in Neurobasal medium supplemented with 2\% B27 and $0.5 \mathrm{~mm}$ glutamine (Invitrogen). Hippocampal neurons at 7 days in vitro (DIV) were transfected with different plasmids plus enhanced green fluorescent protein using calcium phosphate precipitation. The morphometric analysis of neurons was performed using the ImageJ software (National Institutes of Health). 


\section{Results}

\section{Cdk5 is S-nitrosylated in the adult} mouse brain

We first determined whether Cdk5 can be S-nitrosylated by the NO donor. Cdk 5 was overexpressed in HEK $293 \mathrm{~T}$ cells, and the lysate was incubated with the NO donor GSNO or NOC12. We found that Cdk5 was readily S-nitrosylated following treatment with either GSNO or NOC12 (Fig. 1a). Importantly, S-nitrosylated Cdk5 can be detected in the brain. Adult mouse brain lysate was subjected to the biotin switch assay in the presence or absence of ascorbate or biotin-HPDP. S-nitrosylated Cdk5 was only detected in the presence of both ascorbate and biotin-HPDP, suggesting that Cdk5 is S-nitrosylated in vivo (Fig. 1b).

\section{Cdk5 is S-nitrosylated at the Cys83 residue}

As a first step to determine the site(s) of S-nitrosylation on Cdk5, we analyzed its amino acid sequence, which revealed eight cysteine residues (Fig. 2a). We generated mutants of these eight residues, in which each of the cysteine residues alone or in combination was mutated to alanine. HEK $293 \mathrm{~T}$ cells were transfected with wild-type Cdk5 (Cdk5-WT) or its cysteine mutants, and cell lysates were subsequently subjected to biotin switch assay. Treatment of the cell lysate with GSNO resulted in a robust S-nitrosylation of Cdk5-WT. In contrast, mutation of Cys83 alone essentially abolished the S-nitrosylation of Cdk5, indicating that Cys83 is the major site of S-nitrosylation (Fig. $2 b$ ). Other cysteine mutants of Cdk5 could be S-nitrosylated to an extent similar to that observed with Cdk5-WT, consistent with the presence of only one major S-nitrosylation site on Cdk5 at Cys83 (Fig. 2b).

\section{S-nitrosylation of Cdk5 inhibits its kinase activity}

S-nitrosylation regulates protein function primarily through modulating its interaction with the binding partner(s) or its enzymatic activity (Stroissnigg et al., 2007). We found that neither S-nitrosylation of Cdk5 nor mutation of Cys83 on Cdk5 to alanine results in any change in the association between C $\mathrm{dk} 5$ and p35 (Fig. 3a), consistent with the observation that Cys83 is not located in the region responsible for the interaction. Interestingly, Cys83 residue is one of the amino acid residues located in the ATPbinding pocket of $\mathrm{Cdk} 5$, raising the interesting possibility that S-nitrosylation of Cdk5 at Cys83 may perturb the access of the kinase to ATP, resulting in suppression of its activation. To address this possibility, the effect of GSNO incubation on the activity of Cdk5/ p35 was examined using an in vitro kinase assay. Notably, recombinant Cdk5/p35 exhibited reduced kinase activity following incubation with GSNO, indicating that S-nitrosylation of Cdk5 ${ }^{* *} p<0.01$, Student's $t$ test.
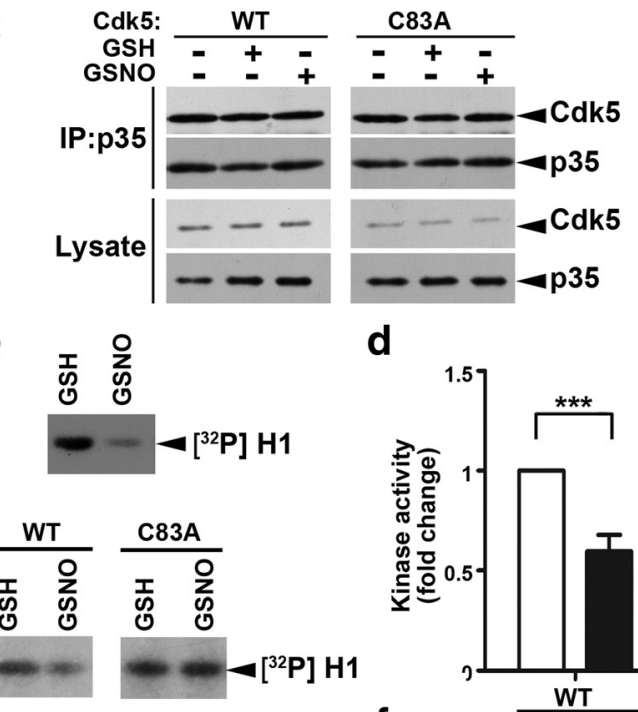

d
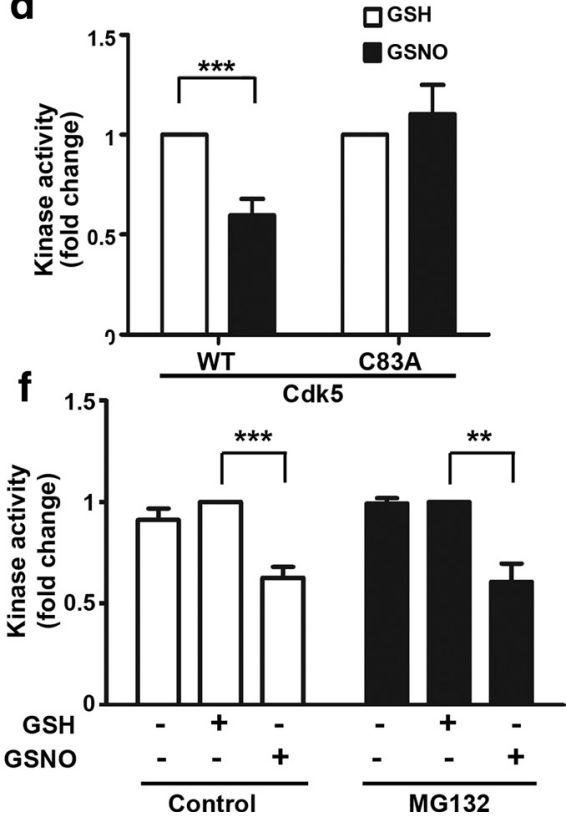

Control

MG132

Figure 3. S-nitrosylation of $C \mathrm{dk} 5$ represses the kinase activity of $C \mathrm{dk} 5 / \mathrm{p} 35 . \boldsymbol{a}$, S-nitrosylation of $\mathrm{Cdk} 5$ does not affect its binding for 30 min and then subjected to the kinase assay using histone $\mathrm{H} 1$ (H1) as substrate $\mathrm{c} d \mathrm{~d}$ S-nitrosylation of $\mathrm{Cdk} 5$ at $\mathrm{Cys} 83$ suppresses Cdk5 kinase activity. S-nitrosylation specifically reduces the kinase activity of p35/Cdk5-WT but not p35/Cdk5-C83A (WT) or its (83A mutant recombinant proteins were incubated with p35-expressing 293T cell lysate. The p35/GST-Cdk5

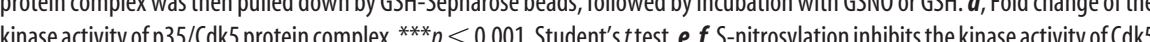
in neurons. Cortical neurons at 8 DIV were exposed to GSNO or GSH for $30 \mathrm{~min}$ in the absence or presence of MG132. Cell lysate was collected and then subjected to Cdk5 kinase assay.f, Fold change of the kinase activity of p35/Cdk5 protein complex. ${ }^{* *} p<0.001$,

suppresses the catalytic activity of Cdk5/p35 ( 70\%) (Fig. $3 b$ ). Next, we examined whether the specific S-nitrosylation of Cdk5 at Cys83 is responsible for the reduced Cdk5/p35 activity. Recombinant Cdk5-WT or its mutant Cdk5-C83A proteins were incubated with the cell lysate prepared from p35-overexpressing HEK 293T cells, followed by exposure to GSH or GSNO. The basal kinase activity of Cdk5-WT/p35 or Cdk5-C83A/p35 was similar, suggesting that $\mathrm{Cdk} 5$ is not $\mathrm{S}$-nitrosylated under basal condition. However, exposure to GSNO reduced the kinase activity of Cdk5-WT/p35, but not that of Cdk5-C83A/p35 (Fig. $3 c, d)$. Similarly, treatment of cortical neurons with GSNO resulted in reduced Cdk5 activity (Fig. $3 e, f$ ). Since a lower p 35 level was observed upon treatment with GSNO (Fig. 3e), cortical neurons were subjected to cotreatment with GSNO and a proteasome inhibitor, MG132, which prevents the degradation of p35 protein. The reduction of Cdk5 activity was similarly observed under this cotreatment condition (Fig. 3e,f), demonstrating that S-nitrosylation of Cdk5 at Cys 83 directly inhibits its kinase activity. 
a

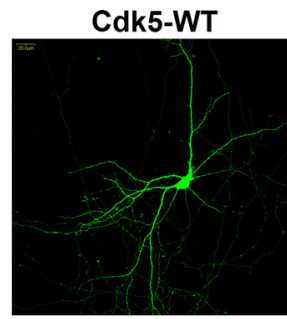

Cdk5-C190A

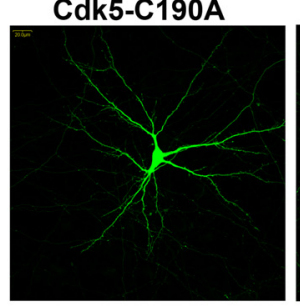

b

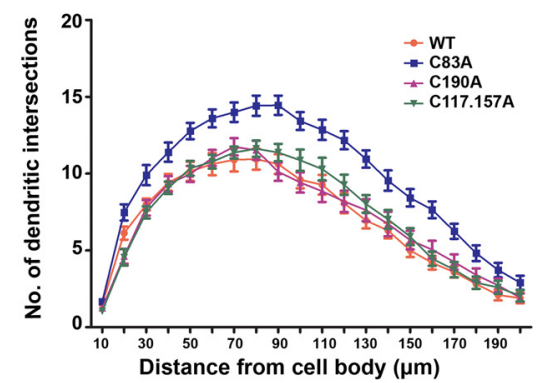

C

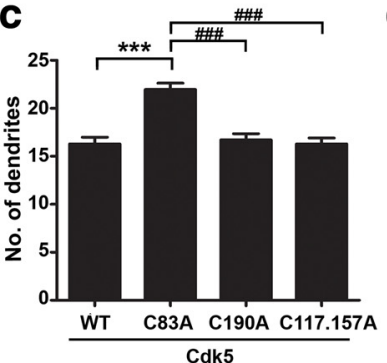

d

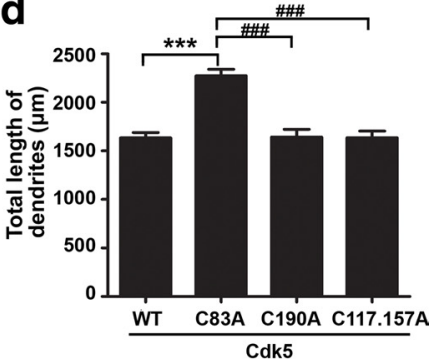

Figure 4. S-nitrosylation of $\mathrm{Cdk} 5$ at the Cys83 residue regulates dendrite development. Cultured hippocampal neurons at 7 DIV were transfected with CDNA expression constructs encoding Cdk5-WT or its cysteine mutants, as indicated, and the morphology of transfected neurons was examined at 14 DIV. $\boldsymbol{a}$, Representative images of hippocampal pyramidal neurons overexpressing Cdk5-WT or its cysteine mutants (C83A; C190A or C117.157A). Scale bar, $20 \mu \mathrm{m}$. $\boldsymbol{b}$, Dendrite development of transfected neurons was quantified by Sholl analysis. Expression of the $C \mathrm{dk} 5$ - $\mathrm{C} 83 \mathrm{~A}$ mutant increases the number of intersections at $30-200 \mu \mathrm{m}$ from soma when compared with that of neurons expressing Cdk-WT or other cysteine mutants. $\boldsymbol{c}, \boldsymbol{d}$, Dendritic branching points and total dendrite length of cultured hippocampal neurons were quantified by image I software. Data shown are mean \pm SEM $(n=20$ neurons for each condition; experiment was repeated three times). ${ }^{* * *} p<0.001$, \#\#\# $<0.001$, Student's $t$ test.

\section{Cdk5 S-nitrosylation regulates dendrite development}

To explore the potential physiological role of Cdk5 S-nitrosylation in neuronal development, the effect of overexpressing Cdk5 or its C83A mutant on neuronal morphology was examined. We have previously demonstrated that Cdk5 knockdown or inhibition of $\mathrm{Cdk} 5$ activity significantly reduces dendrite growth and branching in cultured hippocampal neurons (Fu et al., 2007). In the present study, Cdk5-WT or its cysteine mutants was coexpressed with GFP construct in hippocampal neurons, and the dendrite morphology was examined (Fig. 4a). Compared with the neurons that overexpressed Cdk5-WT, neurons expressing C83A mutant exhibited enhanced dendrite growth, as shown by Sholl analysis (Fig. 4a,b). Quantitative analysis revealed that the total dendritic length and branching points significantly increased in neurons that overexpressed Cdk5-C83A mutant (Fig. 4c,d). These findings suggest that S-nitrosylation of Cdk5 regulates dendrite development, at least in part by modifying the kinase activity of Cdk5.

\section{Discussion}

Extensive studies have demonstrated the important roles of Cdk5 in neurons during development as well as under pathological conditions. Whereas precise control of Cdk5 activity is required for proper neuronal functions, the molecular mechanisms governing its regulation remain elusive. In this study, we identify S-nitrosylation as a novel post-translational regulatory mechanism controlling the kinase activity of Cdk5. S-nitrosylation of Cdk5 at Cys83 results in an inhibition of Cdk5 activity. Importantly, we provide evidence that such S-nitrosylation of Cdk5 regulates dendrite growth and arborization of neurons, implicating a potential physiological role of S-nitrosylated Cdk5. Given the significance of Cdk5 in virtually every aspect of neuronal development and functioning, our findings have opened up a new venue in exploring the pivotal role of S-nitrosylation in regulating the activity of this multifaceted kinase. It will also be of great interest to elucidate the upstream stimuli that modulates Cdk5 activity through S-nitrosylation in the developing or mature CNS.

S-nitrosylation of the target protein at the cysteine residue is known to be facilitated by the flanking acidic and basic residues (Stamler et al., 1997). Interestingly, the Cys83 residue on Cdk5 together with its adjacent residues (Phe-Cys-Asp; FCD) (Fig. 2a) best fits the consensus motif of S-nitrosylation. This sequence motif is conserved in Cdk5 among different species (human, rat, mouse and Xenopus), but not in other members of the Cdk family, suggesting that the regulation of Cdk5 by S-nitrosylation is specific. Interestingly, Cys 83 is one of the critical amino acid residues that form the ATP-binding pocket of Cdk5 (Zhang et al., 2002) and is essential for Cdk5 to form a hydrogen bond with its specific inhibitor roscovitine (Mapelli et al., 2005; Otyepka et al., 2006). It is tempting to speculate that S-nitrosylation of Cys 83 may perturb the binding of ATP to the kinase, thereby resulting in an inhibition of the Cdk5 kinase activity.

$\mathrm{Cdk} 5$ activity has been implicated in the regulation of dendrite growth and spine development, through modulating the cytoskeletal dynamics (Cheung and Ip, 2007). For example, Cdk5 knockdown or inhibition of Cdk5 activity significantly reduces dendrite growth and branching in cultured hippocampal neurons (Fu et al., 2007), and conditional knockout of Cdk5 in mouse cortex results in impaired dendrite development, which is associated with reduced expression of a microtubule-associated protein MAP2 (Ohshima et al., 2007). Since perturbing the S-nitrosylation of Cdk5 enhances dendrite growth and branching in hippocampal neurons (Fig. 4), our findings reveal a new Cdk5dependent regulatory mechanism, further supporting the notion that precise regulation of Cdk5 activity is critical for dendrite development. It is noteworthy that NO signaling has been implicated in regulating the growth of axons and dendrites. For example, S-nitrosylation of histone deacetylase 2 promotes dendrite growth, whereas S-nitrosylation of light chain of microtubuleassociated protein $1 \mathrm{~B}$ leads to retraction of neurites/axons (Stroissnigg et al., 2007; Nott et al., 2008). It is tempting to speculate that S-nitrosylation of Cdk5 and the subsequent reduction of its kinase activity might be specifically induced by extracellular stimuli that trigger dendrite retraction. Studies are in progress to explore the upstream regulators involved in modulating the S-nitrosylation of Cdk5 and its activity in vivo. 
In addition to regulation dendrite development, precise control of Cdk5 activity is also critically involved in other physiological functions, such as synapse formation and plasticity. In this regard, it is noteworthy that excitatory glutamatergic neurotransmission reduces Cdk5 activity through enhancing the proteasome-dependent degradation of p35 (Wei et al., 2005). Interestingly, glutamate stimulation of neurons is able to trigger neuronal nitric oxide synthase activation, and results in protein S-nitrosylation (Bredt and Snyder, 1994; Song et al., 2008), making glutamate a likely candidate that can inhibit Cdk5 activity through S-nitrosylation. Thus, it is possible that S-nitrosylation of Cdk5 also contributes, at least in part, to the reduced kinase activity upon glutamate-induced NMDA receptor activation. It will be of great interest to study whether S-nitrosylation of $\mathrm{Cdk} 5$ plays a regulatory role in glutamate-dependent synaptic plasticity.

\section{References}

Bredt DS, Snyder SH (1994) Nitric Oxide: A Physiologic Messenger Molecule. Annu Rev Biochem 63:175-195.

Cheung ZH, Ip NY (2007) The roles of cyclin-dependent kinase 5 in dendrite and synapse development. Biotechnol J 2:949-957.

Cheung ZH, Fu AK, Ip NY (2006) Synaptic roles of Cdk5: implications in higher cognitive functions and neurodegenerative diseases. Neuron 50:13-18.

Chung KK (2006) Say NO to neurodegeneration: role of S-nitrosylation in neurodegenerative disorders. Neurosignals 15:307-313.

Cruz JC, Tsai LH (2004) A Jekyll and Hyde kinase: roles for Cdk5 in brain development and disease. Curr Opin Neurobiol 14:390-394.

Dhavan R, Tsai LH (2001) A decade of CDK5. Nat Rev Mol Cell Biol 2:749-759.

Fu WY, Chen Y, Sahin M, Zhao XS, Shi L, Bikoff JB, Lai KO, Yung WH, Fu AK, Greenberg ME, Ip NY (2007) Cdk5 regulates EphA4-mediated dendritic spine retraction through an ephexin1-dependent mechanism. Nat Neurosci 10:67-76.

Guix FX, Uribesalgo I, Coma M, Muñoz FJ (2005) The physiology and pathophysiology of nitric oxide in the brain. Prog Neurobiol 76:126-152.

Hisanaga S, Saito T (2003) The regulation of cyclin-dependent kinase 5 activity through the metabolism of p 35 or p $39 \mathrm{Cdk} 5$ activator. Neurosignals 12:221-229.

Kamei H, Saito T, Ozawa M, Fujita Y, Asada A, Bibb JA, Saido TC, Sorimachi H, Hisanaga S (2007) Suppression of calpain-dependent cleavage of the CDK5 activator p 35 to p25 by site-specific phosphorylation. J Biol Chem 282:1687-1694.

Kusakawa G, Saito T, Onuki R, Ishiguro K, Kishimoto T, Hisanaga S (2000) Calpain-dependent proteolytic cleavage of the p35 cyclin-dependent kinase 5 activator to p25. J Biol Chem 275:17166-17172.

Lai KO, Ip NY (2009) Recent advances in understanding the roles of Cdk5 in synaptic plasticity. Biochim Biophys Acta 1792:741-745

Lee MS, Kwon YT, Li M, Peng J, Friedlander RM, Tsai LH (2000) Neurotoxicity induces cleavage of p35 to p25 by calpain. Nature 405:360-364.

Lipton SA, Choi YB, Sucher NJ, Chen HS (1998) Neuroprotective versus neurodestructive effects of NO-related species. Biofactors 8:33-40.

Mapelli M, Massimiliano L, Crovace C, Seeliger MA, Tsai LH, Meijer L,
Musacchio A (2005) Mechanism of CDK5/p25 binding by CDK inhibitors. J Med Chem 48:671-679.

Nott A, Watson PM, Robinson JD, Crepaldi L, Riccio A (2008) S-Nitrosylation of histone deacetylase 2 induces chromatin remodelling in neurons. Nature 455:411-415.

Ohshima T, Hirasawa M, Tabata H, Mutoh T, Adachi T, Suzuki H, Saruta K, Iwasato T, Itohara S, Hashimoto M, Nakajima K, Ogawa M, Kulkarni AB, Mikoshiba K (2007) Cdk5 is required for multipolar-to-bipolar transition during radial neuronal migration and proper dendrite development of pyramidal neurons in the cerebral cortex. Development 134:2273-2282.

Otyepka M, Bártová I, Kríz Z, Koca J (2006) Different mechanisms of CDK5 and CDK2 activation as revealed by CDK5/p25 and CDK2/cyclin A dynamics. J Biol Chem 281:7271-7281.

Patzke H, Tsai LH (2002) Calpain-mediated cleavage of the cyclindependent kinase-5 activator p39 to p29. J Biol Chem 277:8054-8060.

Rahman MA, Senga T, Ito S, Hyodo T, Hasegawa H, Hamaguchi M (2010) S-nitrosylation at cysteine 498 of c-Src tyrosine kinase regulates nitric oxide-mediated cell invasion. J Biol Chem 285:3806-3814.

Reynaert NL, Ckless K, Korn SH, Vos N, Guala AS, Wouters EF, van der Vliet A, Janssen-Heininger YM (2004) Nitric oxide represses inhibitory kappaB kinase through S-nitrosylation. Proc Natl Acad Sci U S A 101:8945-8950.

Saito T, Onuki R, Fujita Y, Kusakawa G, Ishiguro K, Bibb JA, Kishimoto T, Hisanaga S (2003) Developmental regulation of the proteolysis of the p35 cyclin-dependent kinase 5 activator by phosphorylation. J Neurosci 23:1189-1197.

Song T, Hatano N, Kambe T, Miyamoto Y, Ihara H, Yamamoto H, Sugimoto K, Kume K, Yamaguchi F, Tokuda M, Watanabe Y (2008) Nitric oxidemediated modulation of calcium/calmodulin-dependent protein kinase II. Biochem J 412:223-231.

Stamler JS, Toone EJ, Lipton SA, Sucher NJ (1997) (S)NO signals: translocation, regulation, and a consensus motif. Neuron 18:691-696.

Stroissnigg H, Trancíková A, Descovich L, Fuhrmann J, Kutschera W, Kostan J, Meixner A, Nothias F, Propst F (2007) S-nitrosylation of microtubuleassociated protein $1 \mathrm{~B}$ mediates nitric-oxide-induced axon retraction. Nat Cell Biol 9:1035-1045.

Tsang AH, Lee YI, Ko HS, Savitt JM, Pletnikova O, Troncoso JC, Dawson VL, Dawson TM, Chung KK (2009) S-nitrosylation of XIAP compromises neuronal survival in Parkinson's disease. Proc Natl Acad Sci U S A 106:4900-4905.

Wei FY, Tomizawa K, Ohshima T, Asada A, Saito T, Nguyen C, Bibb JA, Ishiguro K, Kulkarni AB, Pant HC, Mikoshiba K, Matsui H, Hisanaga S (2005) Control of cyclin-dependent kinase 5 (Cdk5) activity by glutamatergic regulation of p35 stability. J Neurochem 93:502-512.

Whalen EJ, Foster MW, Matsumoto A, Ozawa K, Violin JD, Que LG, Nelson CD, Benhar M, Keys JR, Rockman HA, Koch WJ, Daaka Y, Lefkowitz RJ, Stamler JS (2007) Regulation of beta-adrenergic receptor signaling by S-nitrosylation of G-protein-coupled receptor kinase 2. Cell 129:511-522.

Yasukawa T, Tokunaga E, Ota H, Sugita H, Martyn JA, Kaneki M (2005) S-nitrosylation-dependent inactivation of Akt/protein kinase B in insulin resistance. J Biol Chem 280:7511-7518.

Zhang J, Luan CH, Chou KC, Johnson GV (2002) Identification of the $\mathrm{N}$-terminal functional domains of Cdk5 by molecular truncation and computer modeling. Proteins 48:447-453. 